\title{
«Quaderni di Filologia e Lingue romanze», Ricerche svolte nell'Università di Macerata, Terza serie, volume 14; Macerata, 1999; 464 pp. + Indice
}

Poiché le linee direttrici del periodico maceratese continuano ad essere quelle dei volumi precedenti, anche la presente recensione segue i principi finora adottati, concentrandosi sui contributi linguistici e/o filologici e abbreviando la presentazione degli altri.

Gabriella Almanza Ciotti (Il cavaliere Perceval /Elementi per una riletturaf) (5-22): ad un'introduzione su Chrétien de Troyes e una breve rassegna dei suoi romanzi segue il resto dell'articolo che si dedica a Perceval.

Uberto Malizia (Il lessico della musica in Galeran de Bretagne: un approccio letterario) (23-46): si esaminano i termini attinenti alla musica (buisine, chalemel e challemelle, cor, harpe e herpe, vielle) facendo risaltare l'importanza della musica in ambienti cortesi (43).

Luca Pierdominici (Con Martial d'Auvergne alla corte del parlamento d'amore: Gli Arrêts d'amour) (47-75): lo studio ci informa sullo scrittore quattrocentesco, sulla sua opera "Sentenze d'amore" e la relativa interpretazione (62-65: sistema verbale). Conclusione (riassunto): 66 . Ci sono 46 note con molto materiale bibliografico e altro.

Maryvonne Baurens (Des derniers actes officiels en occitan-béarnais: La délibération des états de Béarn du 6 mars 1789) (77-105): è uno dei contributi, piuttosto rari nella nostra rivista, di interesse linguistico, e anche storico, perché attesta un momento molto importante della "nazione" [virgolette nel testo] bearnese. Il documento non è meno importante nella storia linguistica dell'idioma locale (81), il quale "demeure, comme l'atteste notre document, une forme symbolique de résistance vis-à-vis de la politique du gouvernement français" (83). Dopo l'introduzione storica (79-84) leggiamo la rassegna dei fatti notevoli di livello grafico, fonetico, sintattico, morfologico e lessicale (84-89) e il riassunto-conclusioni (89-90), poi le 53 note, che non sono soltanto referenze ma contengono anche molto materiale sostanziale (90-96), i criteri di redazione (97-98) ed il testo (99-105). Seguono 17 pagine di riproduzione in facsimile del testo manoscritto, fuori paginazione. Il contributo è di alto valore linguisti$\mathrm{co}$, filologico nonché storico, per la linguistica francese e anche su scala romanza.

Elisabeth Ceaux (Des grives aux loups de Claude Michelet: un roman de la mémoire paysanne) (107-132): presentazione del primo dei 4 romanzi, dedicato alla terra e ai contadini (nel $20^{\circ}$ secolo), una famiglia che "a donc un amour forcené de la terre" (115).

Michela Tognetti (Henri Perruchot e l'Epifanismo) (133-158): articolo sul detto autore, molto produttivo al suo tempo ma poi dimenticato, creatore dell'epifanismo (dottrina che accentua la libertà dell'uomo), scomparso con la morte di Perruchot nel 1967 (154).

Silvia Volpini - Margherita Breccia (Intervista a Roger Bichelberger) (159-179): serie di domande (vocazione di scrittore, ispirazione, motivi ecc.) rivolte allo scrittore 
lorenese (in cui la natura ha una parte predominante, perché in essa l'uomo trova "se stesso e Dio"; 173).

Luigi Banfi (Intorno al cantare di san Giovanni Boccadoro) (181-215): la nota leggenda è qui al centro dell'interesse filologico (184), come risulta dal confronto e dalla minuziosa analisi delle due versioni. Segue il testo conservato alla Biblioteca di Bergamo con (in note) i necessari confronti tra esso e la versione della Braidense di Milano (testo: 203-212; note 213-215).

Rita Monacelli Tommasi (Leggenda di santa Margherita d'Antiochia/Edizione del ms. 1658 della Biblioteca Riccardiana di Firenze/) (217-241): lo studio si riconnette a quello nel num. 12 dei QFLR e consiste di una breve introduzione (219-220), a cui segue il testo (221-232), seguito a sua volta dalle annotazioni (233-240) e dalle note (241).

Annamaria Fabiani ("Il peso del matrimonio". Doti nuziali a Montolmo nel Settecento) (243-278): descrizione degli oggetti appartenenti al corredo nuziale a Montolmo (anche Monte dell'Olmo; antica Pausula, oggi Corridonia), interessante per la terminologia (utensili, abbigliamento, biancheria ecc.) e per quello che vi si scorge sulla posizione della donna, sul livello socio-economico, la dote ecc. In Appendice: la trascrizione dei due documenti con la riproduzione in facsimile dei relativi manoscritti. Le 93 note (271-278) contengono molte spiegazioni e commenti dei termini.

Emilietta Panizza (Léxico exótico en obras del cautiverio español) (279-304): presentazione degli esotismi negli scritti (per lo più di carattere autobiografico; uno di essi e M. de Cervantes) dei prigionieri spagnoli (nel Cinque- e Seicento) in Turchia e alcuni altri paesi musulmani. L'elenco è alquanto modesto: soli 14 termini, da agás (turco aga "oficial del ejército turco") a zerraje (turco seray, saray "serrallo, palacio"); eppure secondo l'autrice "la cantidad de palabras exóticas es, en realidad, copiosa. Hasta el punto de que podría redactarse un diccionario de las glosas presentadas por los propios autores" (299). Parecchio materiale bibliografico si trova in alcune (delle 52) note.

Lorella Maria Rota (I prodromi della Guerra Civile spagnola) (305-327): contributo di ovvio interesse, il quale nel contempo esce dalla solita tematica della rivista, occupandosi della situazione politica che ha portato alla detta guerra (l'inizio degli anni 20, i partiti, il conflitto tra chiesa e liberali, i personaggi italiani /A. De Gasperi, don L. Sturzo, F. Alessandrini/ e i periodici principali /La Civilità Cattolica, L'Osservatore Romano/).

Daniela Cingolani (Il cromatismo nelle Sonatas di Valle-Inclán/III parte/) (329-368) è l'ultima parte dello studio, in cui, analogamente alle prime due, si esaminano i concetti (e i termini) luce-luminosità (brillante/opaca), i colori rosa, grigio, viola, arancione, malva, marrone, e i vari colori insieme. Le "tavole" sono: elementi della natura, oggetti inanimati, corpo umano, anima e $i$ suoi riflessi, abbigliamento e tessuti, animali, esseri soprannaturali, incrociate con le categorie sintattiche (nomi, aggettivi, verbi). Anche in questa terza parte abbondano fini analisi stilistiche. Le note ( 238 in tutto) sono per lo più citazioni, solo l'ultima nota (pp. 367-368) fornisce le indicazioni 
statistiche delle frequenze cromatiche in ciascuna delle Sonatas [secondo il nostro calcolo, ci sono in tutti i testi ben 857 frequenze cromatiche complessive].

Carlos Alberto Cacciavillani (La cultura, l'urbanistica e l'architettura del Modernismo catalano tra la fine del XIX e l'inizio del XX secolo) (369-396): breve introduzione storica, 11 pagine di fotografie (373-383), commenti sugli architetti modernisti (A. Gaudí, L. Domènech i Montaner, Josep Puig i Cadafalch), con uno sguardo sul Medioevo ("unico passato autentico"), 387) e sull'architettura e decorazione islamica (392-394).

José Ramón Fernández de Cano y Martín (La dimensión erótica del léxico taurino) (397-426): si esaminano le metafore (dalla mitologia antica fino ad oggi) tra il linguaggio della tauromachia e quello erotico ("la figura del toro bravo puede encarnar la pulsión sexual masculina en las relaciones eróticas entre un hombre y una mujer", 404). Un buon riassunto si trova alle pp. 414-416, e anche qui alcune (delle 70) note contengono materiale interessante.

Thais A. Fernández (Somiglianze di famiglia: riflessioni sulla traduzione di Mafalda) (427-442): si tratta della traduzione dell'omonimo fumetto argentino, di cui si riportano 8 pagine di strisce con commenti. Il fumetto su Mafalda non è soltanto argentino: infatti, si tratta di "presupposti comuni alle società occidentali, che vanno molto al di lá del segno puramente linguistico" (432).

Daniela Fabiani (Il tempo finzionale della bellezza in Un coro a più voci di Rosa Berti Sabbieti. Note di lettura) (445-455): saggio letterario, dedicato al recente romanzo della scrittrice, il quale si basa sui ricordi ma li inquadra contemporaneamente in un tempo a-temporale, "un tempo nel tempo ma fuori del tempo" (Eliot; 451), un "tempo cronologico e contemporaneamente mitico" (452). Il romanzo è "una narrazione che supera le dialettiche esistenti", "una sintesi di storia letteraria e nel contempo [...] una proposta di nuovi orizzonti verso cui tendere" (455).

Infine, Luca Pierdominici recensisce (459-464) il volume Guillaume de Lorris, Le Roman de la Rose, présentation, traduction inédite, notes, bibliographie, chronologie et index par Jean Dufournet, Paris, GF-Flammarion 1999. La recensione, beninteso positiva, si sofferma dapprima a lungo sulla introduzione dello studioso francese, assumendo un po' le dimensioni di un saggio letterario autonomo; seguono cenni sul testo, la traduzione e le note di Dufournet. In conclusione (464): "Jean Dufournet fa [...] del suo libro un modello di trasparente chiarezza per tutti coloro che vogliono leggere o rileggere il capolavoro della leterattura allegorica medievale in lingua d'oül, dando al contempo un saggio di metodologia e di erudizione".

Gli errori tipografici nel volume recensito sono rari. I «Quaderni di Filologia e Lingue romanze» continuano a presentare le caratteristiche che già conosciamo: bella veste grafica, presenza delle sole tre grandi lingue romanze occidentali, prevalenza assoluta della tematica letteraria sulle altre.

Pavao Tekavčić 